\title{
Focal mechanism solutions of certain earthquakes in Mizoram and its vicinity using P-wave first-motion data
}

\author{
Saitluanga \\ Department of Geology, Pachhunga University College, Aizawl 796001, Mizoram, India \\ Corresponding author: stasailo@gmail.com
}

\begin{abstract}
Determination of focal mechanism solution permits characterization of the earthquake source most effectively with the estimation of fault geometry. Fault geometry is described in terms of the orientation of the fault plane and the direction of slip along the plane. However, the body wave, surface wave and the parameters such as equivalent forces at the source and seismic impulse response etc. are used to determine the orientation of fault plane and the direction of the movement associated with the earthquake. Since the majority of earthquake source processes are either complicated or cannot be observed directly, it has been necessary to find ways to determine source characteristics from seismic wave motion. The source mechanisms through P-wave first-motion data are determined by acquiring data from local seismic network supplemented by phases from the stations at teleseismic distances. Focal mechanism solutions for the events $M>4.0$ are well constrained. Although the uniform distribution of azimuth of a large number of stations is one of the important prerequisites for a reliable focal mechanism solution, this is not the case for the events $(M<4.0)$ originated in $N E R$. This situation worsens in the case of events $M<3.0$. Determinations of focal mechanisms for these events tend to near impossible with first motion P-wave arrival because very less number of stations record these events.
\end{abstract}

Keywords: Focal mechanism, earthquake, fault geometry, P-wave first-motion data.

\section{INTRODUCTION}

Focal mechanism solution using P-wave first-motion data is one of the simplest methods to determine fault geometry. The basic idea of this method is that, the polarity (direction) of the first $\mathrm{P}$-wave arrival varies between seismic stations at different directions from an earthquake (Ligorria and Ammonm, 1999). The first motion is either compression, for the stations located such that material near the fault moves towards the station or dilatation, where the motion is away from the station. Thus, when a P-wave arrives at a seismometer from Earth's interior, a vertical component seismogram records an upward or downward first motion corresponding to either compression or dilatation (Curtis et al., 2009).

The P-wave first-motion data and the azimuth of the recording stations are collected from different stations which forms the prime input to the determination of focal mechanism solution. The focal mechanism solutions of 67 numbers of events form the database for the present study. Most of these solutions are obtained from GCMT (Global Centroid Moment Tensor) database. Few solutions are determined for the present purpose by incorporating more than 45 stations data from ISC Bulletin, RRL-Jorhat and NGRI-Hyderabad seismological bulletin as well.

\section{MATERIALS AND MethodS}

Determination of focal mechanism solution using $\mathrm{P}$ wave first-motion data requires maximum number of seismograms recorded by different stations. As lower magnitude event is recorded by a very few stations, higher magnitude event $(M \geq 4.0)$ is generally preferred for this method. The events having magnitude $M>4.0$ are recorded by more than 45 numbers of seismic stations including the stations at teleseismic distances. The 
$\mathrm{P}$ and $\mathrm{S}$ phases are re-read from the available analogue seismograms obtained from the local seismic stations. These events are relocated based on crustal velocity model of Bhattacharya et al. (2005), using data for relatively larger number of seismic stations with reasonable degree of azimuthally coverage. In this study, a few focal mechanism solutions are determined (as listed in Table 1) utilizing P-wave $1^{\text {st }}$ motion polarities. These are made on the basis of high precision location determination, clear record of $\mathrm{P}$-wave first-motion polarity, high signal to noise ratio and recorded by maximum number of seismic stations.

Primarily, P-wave polarity, azimuth from the source, incident angle with respect to focal depth and epicentral distance in degrees etc. are the input for determination of focal mechanism solution. The P-wave first-motion data are obtained from local stations as well as stations at teleseismic distances. ISC Bulletin provides P-wave first-motion data from stations at teleseismic distances.

The fault geometry is found from distribution of first motion data on a conceptual homogeneous sphere around the focus called focal sphere. In general Wolff's net (Figure 1a) is used to project the geographical position of an observation station to a point where the tangent to the ray at the source intersects the focal sphere. The $\mathrm{P}$-wave ray path leaving the source can be identified by two parameters: the azimuth from the source $\left(f_{s}\right)$ and the ray parameter or take off angle, $\mathrm{i}_{\mathrm{h}}$ (Lay et al., 1995). The azimuth of the direction of the ray from the source and the takeoff angle prescribes a unique path through the earth to a point on the surface and the corresponding portion of the outgoing wave front is designed to reach that point, conveying the initial motion in the associated region of the outgoing wave. Depending on the distribution of P-wave first motions, the zone of compression and dilatations are identified.

Focal mechanisms were determined using the steps as follows:

1. P-wave first-motion data are obtained from the seismograms of each recording station.

2. Azimuth (fs) and the epicentral distance $(\Delta)$ are calculated for known earthquake and recording stations.

3. The take-off angle, $i_{h}$ is obtained from the table prepared by Hodgson (1953) and, Pho and Behe (1972).

4. Using fs and $i_{h}$ the ray position is projected to each station on a lower hemisphere stereographic projection using Wulff's Net. Different symbols are used to indicate the P-wave first motion. Open circles are used to indicate dilatation and filled circles for compression.

5. The first motion data are rotated on the stereo net to find a meridian line that separates compression from dilations. This plane is drawn along with the pole to the plane, which projects at $90^{\circ}$ from the plane.

6. The data plot is rotated to find a second meridian that separates dilatations and compressions and passes through the pole of the first plane.
7. Out of these two nodal planes the fault plane is ascertained based on the local geology inherited in the region.

8. The strike and dip of the fault planes are determined as illustrated in Figure 1b. Rake is calculated from the poles.

9. Pressure (P) and tension ( $T$ ) axis is determined. The maximum compressive strain $P$ is a pole lying on the plane containing the pole of the two fault planes. It lies in the dilatational quadrants $45^{\circ}$ from the two nodal planes. The minimum compressive stress axis, $T$ lies in the compressional quadrant $45^{\circ}$ from the two planes.

10. The intermediate compressive stress axis, also called $B$ axis or null axis, is determined along the line of intersection of the two nodal planes.

\section{RESULTS}

The focal mechanism solutions of 67 numbers of events form the database for the present study (Figure 2). Most of these solutions are obtained from GCMT (Global Centroid Moment Tensor) database. Few solutions are determined for the present purpose by incorporating more than 45 stations data from ISC Bulletin, RRLJorhat and NGRI-Hyderabad seismological bulletin as well.Distribution of dataset for the events depicting the percentage of faulting type is shown in Table 2. It is observed that the region is dominated by existence of higher percentage of strike-slip faulting. Table 1 indicates the events that comprises of focal mechanism solutions determined through first motion $\mathrm{P}$-wave polarities and CMT (Centroid Moment Tensor) solution. Four solutions (black and white) are obtained through waveform inversion (Figure 2). Fault plane solutions for the events associated with the region are shown separately in Figure 3. A depth section of the beachball representing Figure $3 a$ is shown in Figure $3 \mathrm{~b}$. Figure $3 \mathrm{c}, \mathrm{d}, \mathrm{e}, \mathrm{f}$ indicate the associated nodal plane, $\mathrm{P}$-axis orientation, $\mathrm{T}$-axis orientation and both $\mathrm{P}$ - and T-axis orientation along the eastern part of the Surma valley. Most of the nodal planes are oriented along North-South while P-axis orientation is found to be along north of north-east.

The beachball presentation in Figure $3 b$ for regions in the eastern part appears quite significant when a depth level of $41-60 \mathrm{~km}$ is considered. The events occurring within this depth range are characterized by the regime of dip-slip and thrust type of focal mechanisms. Figure $3 \mathrm{c}$ shows nodal planes of the focal mechanism solutions of the events associated with the study regions. Most of the solutions are characterized by thrust and strike slip fault.

It may be noted that in later figures as mentioned, the definition of the azimuth as would be seen in a map view is retained while the center of each line corresponds to the position in depth, of the solutions. An important observation is that $\mathrm{P}$-axis orientations are pre- 
(a)

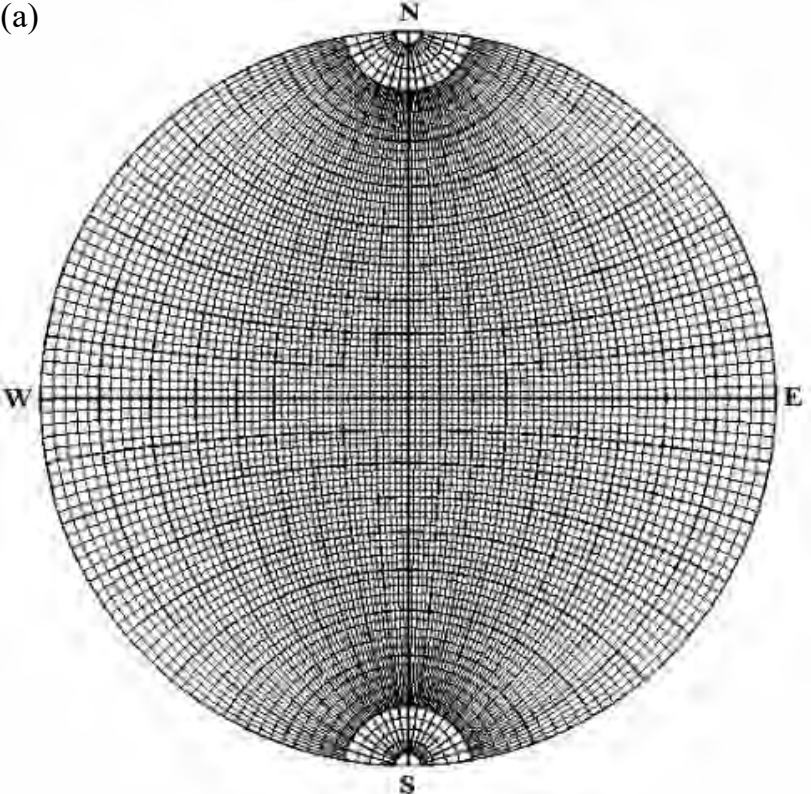

(b)

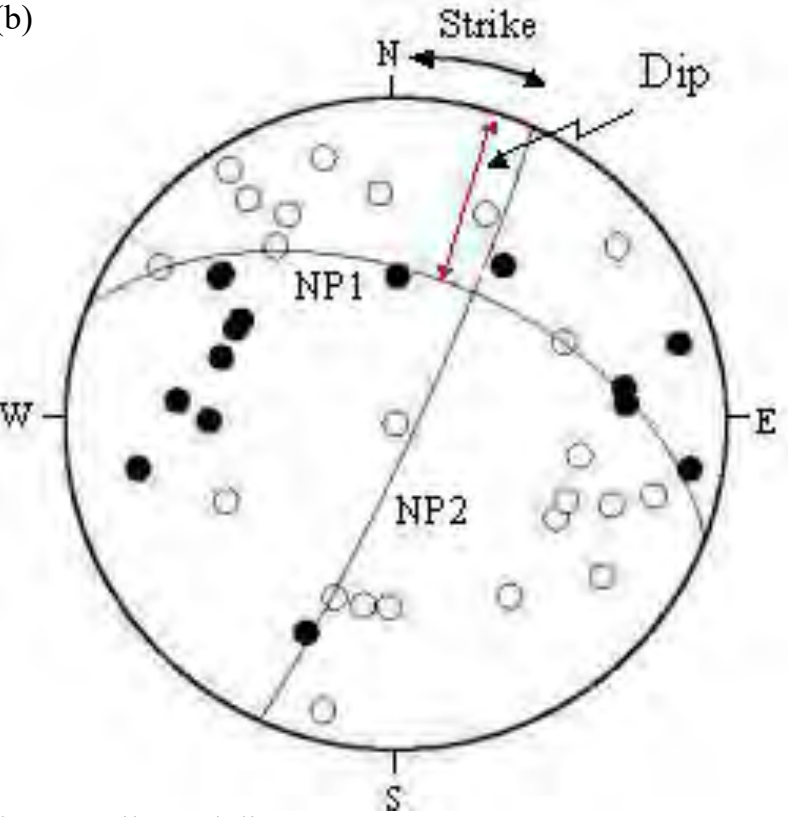

Figure 1: (a) Wulff's net; (b) an example showing the nodal planes, strike and dip.

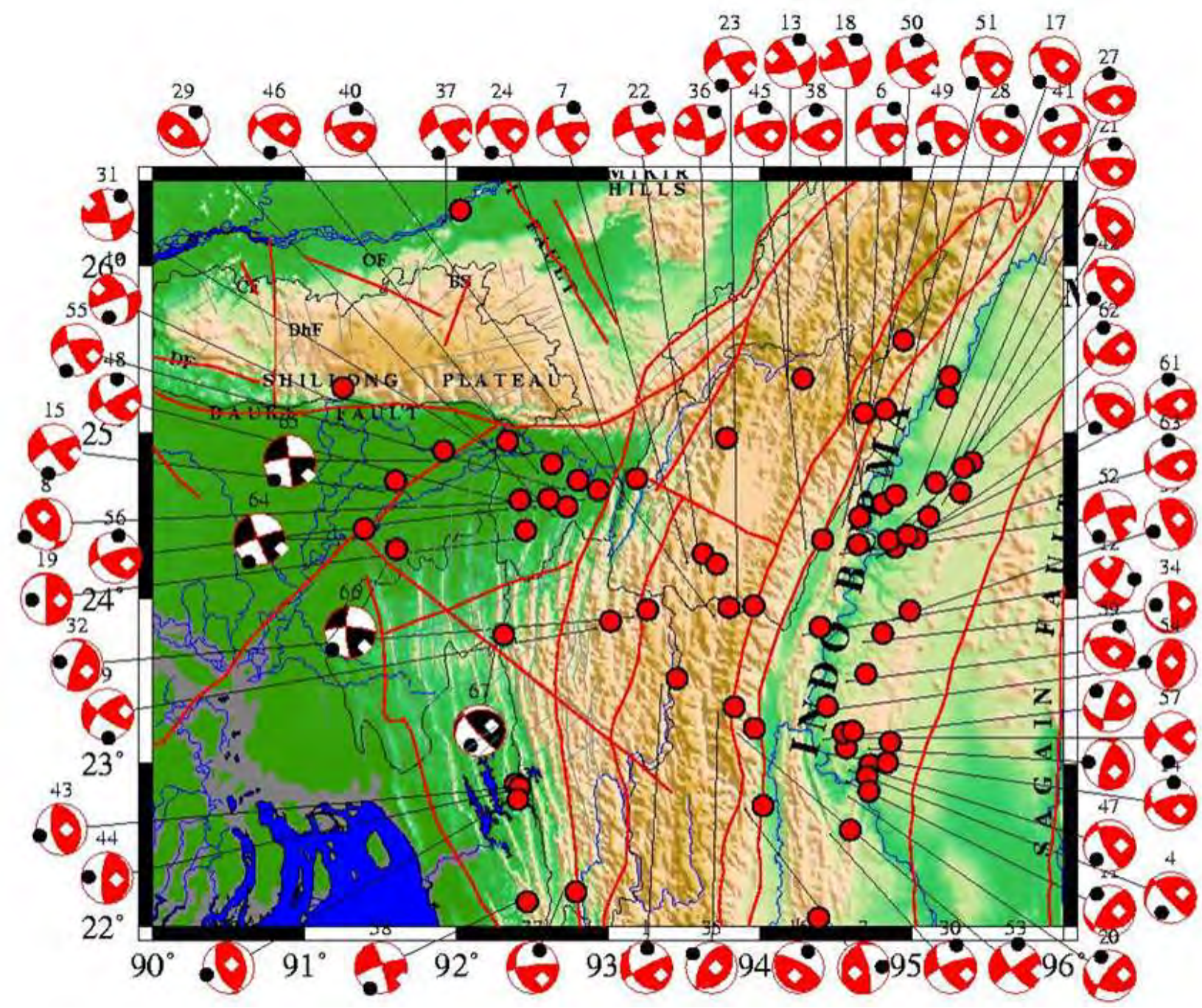

Figure 2: Beachball representation of the focal mechanism solutions in the Surma valley and its vicinity. The black colored beachball represent the focal mechanism solutions through waveform inversion whereas red coloured beachball represent the focal mechanism solution obtained from P-wave first motion. 
Table 1: Parameters of the focal mechanism solutions for earthquakes in and around Surma Valley used in this study (Source: CMT and FMS determined in this study).

\begin{tabular}{|c|c|c|c|c|c|c|c|c|c|c|c|c|}
\hline Year & Month & Day & $\mathbf{H r}$ & Min & Sec & Lat & Long & Depth & Mag & Strike & DIP & SLIP \\
\hline 1977 & 10 & 13 & 11 & 32 & 9.3 & 23.48 & 93.35 & 61 & 5.2 & 145 & 41 & 171 \\
\hline 1978 & 2 & 23 & 23 & 18 & 33.2 & 23.09 & 94.72 & 105 & 5.1 & 331 & 31 & 44 \\
\hline 1980 & 11 & 20 & 18 & 14 & 11.7 & 22.72 & 93.9 & 33 & 5.3 & 208 & 19 & 131 \\
\hline 1981 & 5 & 1 & 4 & 8 & 10.4 & 22.96 & 94.59 & 101 & 5 & 247 & 22 & 21 \\
\hline 1983 & 8 & 23 & 12 & 12 & 16.9 & 24.57 & 95.2 & 126 & 5.1 & 297 & 44 & 58 \\
\hline 1983 & 8 & 30 & 10 & 39 & 27.3 & 25.07 & 94.72 & 63 & 5.6 & 161 & 65 & 159 \\
\hline 1984 & 5 & 6 & 15 & 19 & 11.4 & 24.22 & 93.52 & 33 & 5.7 & 157 & 69 & 161 \\
\hline 1984 & 12 & 30 & 23 & 33 & 39.1 & 24.6 & 92.84 & 33 & 5.6 & 352 & 46 & 123 \\
\hline 1986 & 2 & 8 & 0 & 28 & 53.9 & 23.82 & 92.92 & 33 & 5.2 & 224 & 62 & 15 \\
\hline 1986 & 2 & 19 & 17 & 34 & 24.6 & 25.2 & 91.17 & 18 & 5.3 & 340 & 50 & 180 \\
\hline 1986 & 4 & 26 & 0 & 25 & 59.6 & 22.89 & 94.57 & 115 & 4.9 & 87 & 30 & 136 \\
\hline 1986 & 7 & 26 & 20 & 24 & 48.5 & 23.78 & 94.28 & 33 & 5.1 & 130 & 72 & 15 \\
\hline 1987 & 5 & 18 & 1 & 53 & 51 & 25.26 & 94.18 & 50 & 5.6 & 67 & 68 & 14 \\
\hline 1987 & 8 & 24 & 9 & 24 & 40.5 & 23.06 & 94.44 & 92 & 5.1 & 135 & 42 & 144 \\
\hline 1988 & 2 & 6 & 14 & 50 & 42.7 & 24.65 & 91.52 & 15 & 5.8 & 239 & 76 & 9 \\
\hline 1988 & 7 & 3 & 8 & 19 & 18.9 & 22.05 & 94.25 & 86 & 5.2 & 133 & 18 & 104 \\
\hline 1988 & 8 & 6 & 0 & 36 & 24.6 & 25.14 & 95.12 & 92 & 6.8 & 284 & 45 & 55 \\
\hline 1989 & 4 & 3 & 19 & 39 & 35.1 & 25.05 & 94.58 & 93 & 5.1 & 71 & 81 & 8 \\
\hline 1989 & 4 & 13 & 7 & 25 & 36.6 & 24.36 & 92.37 & 33 & 5.2 & 291 & 6 & 20 \\
\hline 1989 & 7 & 15 & 0 & 9 & 16 & 22.8 & 94.58 & 108 & 5.4 & 111 & 44 & 159 \\
\hline 1990 & 1 & 9 & 18 & 51 & 28.9 & 24.75 & 95.28 & 118 & 6.1 & 140 & 32 & 139 \\
\hline 1991 & 5 & 11 & 2 & 15 & 24.1 & 24.16 & 93.61 & 63 & 5.1 & 159 & 77 & 171 \\
\hline 1991 & 12 & 4 & 3 & 27 & 24 & 23.91 & 93.85 & 70 & 5.2 & 245 & 68 & 11 \\
\hline 1991 & 12 & 20 & 2 & 6 & 5.7 & 24.67 & 93.09 & 47 & 5.5 & 258 & 54 & 30 \\
\hline 1992 & 4 & 15 & 1 & 32 & $\begin{array}{l}9.8 \\
\end{array}$ & 24.3 & 94.91 & 116 & 5.6 & 281 & 45 & 56 \\
\hline 1993 & 4 & 1 & 16 & 30 & 11.2 & 23.15 & 94.42 & 107 & 5.3 & 99 & 44 & 169 \\
\hline 1994 & 8 & 8 & 21 & 8 & 31.9 & 24.72 & 95.22 & 127 & 6 & 111 & 34 & 120 \\
\hline 1995 & 5 & 9 & 9 & 54 & 20.1 & 25.26 & 95.14 & 92 & 5.2 & 142 & 33 & 118 \\
\hline 1996 & 11 & 19 & 0 & 12 & 18.6 & 24.5 & 92.64 & 50 & 5.5 & 102 & 34 & 59 \\
\hline 1997 & 4 & 14 & 17 & 53 & 33.1 & 22.57 & 94.46 & 97 & 4.9 & 150 & 61 & 165 \\
\hline 1997 & 5 & 8 & 2 & 53 & 14.7 & 24.89 & 92.25 & 35 & 5.6 & 78 & 68 & 4 \\
\hline 1997 & 7 & 31 & 15 & 59 & 37 & 23.89 & 93.16 & 33 & 5.5 & 330 & 16 & 40 \\
\hline 1997 & 11 & 21 & 11 & 23 & 6.3 & 22.21 & 92.7 & 54.4 & 5.9 & 163 & 37 & 168 \\
\hline 1998 & 10 & 16 & 0 & 5 & 34.3 & 23.74 & 94.68 & 101.2 & 5.2 & 86 & 12 & 180 \\
\hline 1999 & 2 & 22 & 11 & 37 & 49.4 & 23.31 & 93.72 & 65 & 5.1 & 47 & 35 & 103 \\
\hline 1999 & 4 & 5 & 22 & 32 & 56.5 & 24.91 & 93.68 & 66.4 & 5.3 & 86 & 62 & 16 \\
\hline 1999 & 10 & 5 & 17 & 4 & 44.9 & 26.26 & 91.93 & 33 & 5.3 & 244 & 68 & 12 \\
\hline 2000 & 7 & 2 & 4 & 27 & 57 & 24.51 & 94.69 & 82.5 & 5 & 133 & 37 & 155 \\
\hline 2000 & 10 & 11 & 9 & 42 & 9.3 & 23.87 & 94.86 & 116.5 & 5.4 & 343 & 14 & 98 \\
\hline 2001 & 3 & 3 & 22 & 55 & 59.2 & 23.9 & 93.69 & 55.3 & 5.2 & 140 & 40 & 141 \\
\hline 2001 & 4 & 10 & 22 & 8 & 15.5 & 24.63 & 95.04 & 113.4 & 5 & 8 & 34 & 28 \\
\hline 2001 & 8 & 12 & 1 & 57 & 59.7 & 24.43 & 94.99 & 121.4 & 5 & 285 & 40 & 48 \\
\hline 2003 & 7 & 26 & 23 & 18 & 18 & 22.85 & 92.31 & 10 & 5.5 & 338 & 32 & 82 \\
\hline 2003 & 7 & 27 & 12 & 7 & 29.4 & 22.83 & 92.34 & 10 & 5.2 & 2 & 16 & 88 \\
\hline 2004 & 10 & 8 & 21 & 48 & 5.4 & 24.3 & 94.3 & 69.3 & 4.9 & 139 & 46 & 147 \\
\hline 2004 & 12 & 9 & 8 & 49 & 0.2 & 24.76 & 92.54 & 34.7 & 5.5 & 243 & 42 & 32 \\
\hline 2005 & 1 & 18 & 3 & 2 & 55.1 & 22.97 & 94.7 & $\begin{array}{l}104.7 \\
\end{array}$ & 5 & 271 & 28 & 31 \\
\hline 2005 & 2 & 15 & 11 & 15 & 11.7 & 24.55 & 92.52 & 35.2 & 5.1 & 145 & 52 & 174 \\
\hline 2005 & 2 & 15 & 13 & 5 & 49.8 & 24.43 & 94.54 & 69.7 & 5.2 & 276 & 61 & 28 \\
\hline 2005 & 3 & 25 & 13 & 34 & 40 & 25.48 & 94.84 & 67.2 & 5.3 & 151 & 65 & 176 \\
\hline 2005 & 9 & 18 & 7 & 25 & 59.5 & 24.56 & 94.78 & 84 & 5.6 & 271 & 54 & 38 \\
\hline 2006 & 3 & 2 & 17 & 16 & 60 & 24.27 & 94.53 & 77.9 & 4.9 & 251 & 70 & 5 \\
\hline 2006 & 3 & 25 & 20 & 13 & 30.7 & 23.18 & 93.85 & 61 & 5 & 142 & 76 & 176 \\
\hline 2006 & 5 & 11 & 17 & 22 & 54.1 & 23.31 & 94.32 & 48.3 & 5.7 & 15 & 42 & 102 \\
\hline 2006 & 8 & 12 & 20 & 46 & 11.3 & 24.66 & 92.71 & 42.8 & 4.9 & 255 & 61 & 20 \\
\hline 2006 & 11 & 10 & 13 & 21 & 24.3 & 24.54 & 92.33 & 42.5 & 4.9 & 141 & 46 & 154 \\
\hline 2007 & 5 & 7 & 5 & 58 & 33 & 23.16 & 94.48 & 93.7 & 4.6 & 229 & 69 & 13 \\
\hline
\end{tabular}




\begin{tabular}{|c|c|c|c|c|c|c|c|c|c|c|c|c|}
\hline 2006 & 11 & 10 & 13 & 21 & 24.3 & 24.54 & 92.33 & 42.5 & 4.9 & 141 & 46 & 154 \\
\hline 2007 & 5 & 7 & 5 & 58 & 33 & 23.16 & 94.48 & 93.7 & 4.6 & 229 & 69 & 13 \\
\hline 2007 & 11 & 7 & 7 & 10 & 22 & 22.15 & 92.39 & 28.7 & 5.1 & 251 & 81 & -7 \\
\hline 2007 & 12 & 7 & 6 & 56 & 33.6 & 23.5 & 94.57 & 113.3 & 4.8 & 135 & 42 & 120 \\
\hline 2008 & 1 & 12 & 22 & 44 & 47.4 & 22.76 & 92.33 & 33.8 & 5 & 353 & 35 & 104 \\
\hline 2009 & 8 & 11 & 21 & 43 & 49.8 & 24.25 & 94.77 & 115 & 5.4 & 119 & 45 & 139 \\
\hline 2009 & 9 & 3 & 19 & 51 & 10.5 & 24.29 & 94.73 & 115.5 & 5.7 & 114 & 46 & 152 \\
\hline 2009 & 12 & 29 & 9 & 1 & 55.7 & 24.32 & 94.85 & 127.3 & 5.6 & 123 & 41 & 144 \\
\hline
\end{tabular}

Table 2: Distribution of data set (63 earthquakes) used in the study.

\begin{tabular}{|l|c|c|c|}
\hline Subset & Proportion of total set (per cent) & Average magnitude & Average depth (km) \\
\hline Total & 63 & 5.3 & 70 \\
\hline Reverse & 47.62 & 5.4 & 80 \\
\hline Strike-Slip & 52.38 & 5.3 & 64 \\
\hline
\end{tabular}

Table 3: Parameters of the focal mechanism solutions for earthquakes in the eastern part of Surma valley used in this study (Source : CMT and FMS determined in this study).

\begin{tabular}{|c|c|c|c|c|c|c|c|}
\hline Year & Lat & Long & Depth & Mag & Strike & Dip & Slip \\
\hline 19920521 & 22.98 & 93.34 & 13 & 3.6 & 45 & 65 & 15 \\
\hline 19771013 & 23.27 & 93.16 & 60.8 & 5.4 & 145 & 41 & -171 \\
\hline 19910511 & 23.42 & 93.25 & 73.6 & 5.4 & 159 & 77 & 171 \\
\hline 19860208 & 23.79 & 93.09 & 33 & 5.4 & 224 & 62 & -15 \\
\hline 19890413 & 24.25 & 91.71 & 33 & 5.4 & 291 & 6 & 20 \\
\hline 20010418 & 24.19 & 91.65 & 56 & 3.9 & 330 & 40 & 175 \\
\hline 19681227 & 24.12 & 91.61 & 29 & 5.2 & 140 & 72 & 138 \\
\hline 19880206 & 24.05 & 91.66 & 31 & 5.8 & 239 & 76 & 9 \\
\hline 19710602 & 23.71 & 91.66 & 46 & 5 & 119 & 36 & 90 \\
\hline 19771013 & 23.48 & 93.35 & 61 & 5.2 & 145 & 41 & 171 \\
\hline 19860208 & 23.82 & 92.92 & 33 & 5.2 & 224 & 62 & 15 \\
\hline 19890413 & 24.36 & 92.37 & 33 & 5.2 & 291 & 6 & 20 \\
\hline 20061110 & 24.54 & 92.33 & 42.5 & 4.9 & 141 & 46 & 154 \\
\hline
\end{tabular}

dominantly north north-easterly directed. The direction of the compressional axis long the respective fault indicate the prevailing stress condition of the region. As thrust and strike-slip mechanisms dominate, these two state of mechanisms reflect $\mathrm{N}-\mathrm{S}$ compression rather than E-W extension.

\section{DISCUSSION}

The source mechanisms through $\mathrm{P}$-wave first-motion data are determined by acquiring data from local seismic network supplemented by phases from the stations at teleseismic distances. Focal mechanism solutions for the events $M>4.0$ are well constrained because of availability of required number of $\mathrm{P}$-wave first motions. The uniform distribution of azimuth of a large number of stations is one of the important prerequisites for a reliable focal mechanism solution. However, this is not the case for the events $(M<4.0)$ originated in NER. In case of these events estimated focal mechanism solutions are not well constrained due to sparse distribution of recording sta- tions. This situation worsens in the case of events $M<3.0$. Determinations of focal mechanisms for these events tend to near impossible with first motion P-wave arrival because very less number of stations record these events. However, basic limitation in the study of focal mechanism through P-wave first-motion method for shallow focus earthquake arises by virtue of the very limited coverage of focal sphere by the observation data. The coverage improves with the increase in depth of focus. These constraints are observed by various researchers (Khattri, 1973; Chandra, 1978). The focal mechanism solution using $\mathrm{P}$-wave first-motion data use only a fraction of information contained in body waves. On the contrary the estimation of seismic moment and moment magnitudes are not possible.

\section{REFERENCES}

Bhattacharya, P.M., Pujol, J., Majumdar, R.K., Kayal, J.R. (2005). Relocation of earthquakes in the northern region using joint hypocentre determination method. 
Current Science, 89, 1404-1413.

Chandra, U. (1978). Seismicity, earthquake mechanisms and tectonics along the Himalayan mountain range and vicinity. Physics of the Earth and Planetary Interiors, 16, 109-131.

Curtis, A., Nicolson, H., Halliday, D., Trampert, J., Baptie, B. (2009). Virtual seismometers in the subsurface of the Earth from seismic interferometry. Nature Geoscience, 2(10), 700.

Hodgson, J.H., Storey, R.S. (1953). Tables extending Byerly's fault plane technique to earthquakes of any focal depth. Bulletin of the Seismological Society of America, 43, 49-61.
Khattri, K. (1973). Earthquake focal mechanism studies A review. Earth Science Review, 9, 19-63.

Lay, T., Wallace, T.C. (1995). M odern Global Seismology. Academic Press, New York, USA, p. 521.

Ligorria, J. P., Ammon, C. J. (1999). Iterative deconvolution and receiver-function estimation. Bulletin of the seismological Society of America, 89(5), 1395-1400.

Pho, T.P., Behe, L. (1972). Extended distances and angle of incidence of P-waves. Bulletin of the Seismological Society of America, 62, 885-902. 